\title{
Molecular Characterization and Functional Analysis of Two Petunia PhEILs
}

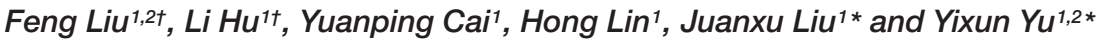 \\ ${ }^{1}$ Guangdong Key Laboratory for Innovative Development and Utilization of Forest Plant Germplasm, College of Forestry and \\ Landscape Architecture, South China Agricultural University, Guangzhou, China, ${ }^{2}$ College of Horticulture, South China \\ Agricultural University, Guangzhou, China
}

\section{OPEN ACCESS}

Edited by:

Antonio Ferrante,

University of Milan, Italy

Reviewed by:

Clay Carter

University of Minnesota, USA

Chi-Kuang Wen,

Shanghai Institutes for Biological

Sciences (CAS), China

*Correspondence:

Juanxu Liu

juanxuliu@scau.edu.cn

Yixun Yu

yuyixun@scau.edu.cn

tThese authors have contributed equally to this work.

Specialty section:

This article was submitted to Plant Physiology,

a section of the journal

Frontiers in Plant Science

Received: 21 March 2016

Accepted: 12 October 2016 Published: 01 November 2016

Citation:

Liu F, Hu L, Cai Y, Lin H, Liu J and Yu Y (2016) Molecular Characterization and Functional Analysis of Two Petunia PhEILs.

Front. Plant Sci. 7:1606. doi: 10.3389/fpls.2016.01606
Ethylene plays an important role in flower senescence of many plants. Arabidopsis ETHYLENE INSENSITIVE3 (EIN3) and its homolog EIL1 are the downstream component of ethylene signaling transduction. However, the function of ElLs during flower senescence remains unknown. Here, a petunia ElL gene, PhElL2, was isolated. Phylogenetic tree showed that PhElL1, whose coding gene is previously isolated, and PhEIL2 are the homologs of Arabidopsis AtEIL3 and AtEIL1, respectively. The expression of both PhEIL1 and PhEIL2 is the highest in corollas and increased during corolla senescence. Ethylene treatment increased the mRNA level of PhElL1 but reduced that of PhEIL2. VIGS-mediated both PhEIL1 and PhEIL2 silencing delayed flower senescence, and significantly reduced ethylene production and the expression of PhERF3 and PhCP2, two senescence-associated genes in petunia flowers. The PhElL2 protein activating transcription domain is identified in the 353-612-amino acids at C-terminal of PhEIL2 and yeast two-hybrid and bimolecular fluorescence complementation assays show that PhEIL2 interacts with PhEIL1, suggesting that PhEIL1 and PhEIL2 might form heterodimers to recognize their targets. These molecular characterizations of PhEIL1 and PhEIL2 in petunia are different with those of in Vigna radiata and Arabidopsis.

Keywords: petunia, VIGS, flower senescence, ethylene signaling, EIL

\section{INTRODUCTION}

Plant hormone ethylene plays an important role in plant growth and development (Abeles et al., 1992). Ethylene perception is mediated by ETR1 family (Chang et al., 1993; Hua et al., 1995, 1998; Schaller and Bleecker, 1995). CONSTITUTIVE TRIPLE RESPONSE 1 (CTR1), a Raf-like Ser/Thr protein kinase, functions in downstream of receptors and in upstream of the central regulator ETHYLENE-INSENSITIVE2 (EIN2) (Kieber et al., 1993; Alonso et al., 1999). EIN2 is phosphorylated by CTR1 to trigger its endoplasmic reticulum (ER)-to-nucleus translocation and to control ethylene signaling from the ER membrane to the nucleus (Ju et al., 2012; Qiao et al., 2012). The C-terminal end of EIN2 (CEND) is thought to participate in signaling output, as ectopic expression of this domain alone can partially activate ethylene responses (Alonso et al., 2003; Wen et al., 2012). The CEND of EIN2 can be phosphorylated by the receptors-activated CTR1 in the absence of ethylene and phosphorylation-regulated proteolytic processing of EIN2 triggers its ERto-nucleus translocation (Ju et al., 2012; Qiao et al., 2012). Inhibition of CTR1 upon ethylene perception is a signal for cleavage and nuclear localization of the EIN2 C terminus to stabilize EIN3 protein (Wen et al., 2012; Ji and Guo, 2013). 
In Arabidopsis, EIN3 and three related EIN3-LIKE (EIL1, EIL2, and EIL3) proteins were shown to possess amino acid sequence similarity and conserved structural features for nuclearlocalized transcription factors (Chao et al., 1997). The ethylene signal is transmitted to the EIN3 family of transcription factors, which have been shown to act as a transcriptional activator and bind to the primary ethylene-response element present in the promoter of the ethylene-responsive ERF1 gene (Chao et al., 1997; Solano et al., 1998). One found that the control of EIN3 degradation is important to regulation of ethylene signaling transduction (Yanagisawa et al., 2003). Two F-box proteins, EBF1 and EBF2 interact with EIN3 and EIL1 (Guo and Ecker, 2003; Potuschak et al., 2003), and disruption of either EBF1 or $E B F 2$ leads to the increase of EIN3 protein levels and induces a hypersensitivity to ethylene. The ebf1 ebf2 double mutant results in a large accumulation of EIN3 proteins and causes a constitutive ethylene response phenotype (Gagne et al., 2004).

Recently, another mechanism of EIN2-mediated ethylene signaling was reported in Arabidopsis ( $\mathrm{Li}$ et al., 2015). The translational repression of $E B F 1$ and EBF2 transcription is imposed by EIN2. The EIN2-directed translational repression is mediated by the EBF1/2 $3^{\prime}$ UTRs and multiple poly-uridylates (PolyU) motifs are identified as functional cis elements of $3^{\prime}$ UTRs (Li et al., 2015).

Ethylene production is increased during flower senescence in many flowers, including petunia, often as the model system for studying the biological bases of flower senescence (Borochov and Woodson, 1989). However, the function of petunia EILs during flower senescence is not well known.

In petunia EIL family, PhEIL1 (accession no. Y353248) has been identified and its expression in response ethylene treatment was reported (Shibuya et al., 2004). Here, another full-length cDNA of petunia EIL gene, PhEIL2, was cloned. PhEIL1 and PhEIL2 expression profile was established in different petunia tissues, at various stages of flower senescence and in response to ethylene. VIGS-mediated both PhEIL1 and PhEIL2 silencing delayed flower senescence in petunia and reduced the expression of two senescence-associated genes. Yeast two-hybrid ( $\mathrm{Y} 2 \mathrm{H})$ and bimolecular fluorescence complementation (BiFC) assays showed that PhEIL2 interacts with PhEIL1.

\section{MATERIALS AND METHODS}

\section{Plant Material}

Petunia (Petunia hybrida 'Ultra') plants were grown under normal greenhouse conditions $\left(22^{\circ} \mathrm{C}, 14\right.$-h light/10-h dark). Flowers were emasculated 1 day before flowers were fully open to prevent self-pollination. Eight to ten petunia flowers were harvested at anthesis stages and placed in distilled water for further processing. Corollas were collected from petunias at $0,1,2,3,4,5,6,7$, and 8 days after flower opening. Stems, leaves and roots were collected from plants at the vegetative stage when the plants were about $10 \mathrm{~cm}$ in height. These tissues were firstly frozen in liquid nitrogen and then stored at $-80^{\circ} \mathrm{C}$. All experiments were performed at least three times.

\section{RNA Extraction and RT-PCR}

RNA extraction and RT-PCR was performed according to the previous protocols (Liu et al., 2010). The RNA content was determined spectrophotometrically. One microgram of total RNA was reverse transcribed at $42^{\circ} \mathrm{C}$ for $1 \mathrm{~h}$ in a final volume of $20 \mu \mathrm{l}$ containing reaction buffer, $20 \mathrm{mmol}^{-1} \mathrm{DTT}, 0.5 \mathrm{mmol}^{-1}$ dNTP, $1 \mu \mathrm{g}$ Oligo (dT) 15 and reverse transcriptase (AMV, Promega, USA) according to the manufacturer's instructions.

\section{Cloning of the Petunia PhEIL2 Gene}

The partial sequence of PhEIL2 was obtained by to the previously described approach (Yang et al., 2009). In brief, TBLASTN analysis against the Genebank EST database ${ }^{1}$ with AtEIN3 and AtEIL1 identified one petunia clone, FN029455, which encodes putative protein displaying high homology with AtEIN3 and AtEIL1, respectively.

The remaining $5^{\prime}$ and $3^{\prime}$ cDNA sequences of PhEIL2 were cloned by rapid-amplification of cDNA ends (RACE) with the forward primer 5'CTATCCTGATCGCTGCCCACCT3' and revered primer $5^{\prime}$ TCTCCAATGGAAATCTTCTCTG3' (Liu et al., 2010).

\section{Sequence Analysis}

The neighbor-joining tree at amino acid level was drawn by DNAMAN software. The reliability of each branch of the tree was assessed using 1,000 bootstrap replications. Identity search for nucleotides and translated amino acids was carried out using National Center for Biotechnology Information (NCBI) BLAST network server ${ }^{2}$.

\section{Ethylene Measurements}

Petunia flowers were treated with ethylene according to previously described protocols (Tan et al., 2014). To measure ethylene production, corollas of each individual flower were collected and placed in a $200 \mathrm{ml}$ airtight container according to the method of Liu et al. (2010). Thus, to avoid the contamination of wound-induced ethylene, the containers were capped and incubated at $25^{\circ} \mathrm{C}$ for $1 \mathrm{~h}$. Next, a $2 \mathrm{ml}$ sample of head-space gas was withdrawn using a gas-tight hypodermic syringe and was injected into a gas chromatograph (GC 17A, Shimadzu, Kyoto, Japan) to measure the ethylene concentration. The gas chromatograph was equipped with a flame ionization detector and an activated alumina column. All measurements were performed in five replicates.

\section{Quantitative Real-Time PCR Assays}

Total RNA extracted from various tissues was digested with DNAase I and then reverse transcription (RT) was performed according to the kit instruction (TaKaRa, China). PCR analysis was carried out with the cDNA as a template. Specific primer design was performed using the sequences obtained for PhEIL1, PhEIL2, PhERF3, and PhCP2. The petunia Actin (accession no. FN014209) genes were used as the internal reference gene

\footnotetext{
${ }^{1}$ http://www.ncbi.nlm.nih.gov

${ }^{2}$ https://blast.ncbi.nlm.nih.gov/Blast.cgi
} 
to quantify the cDNA abundance (Mallona et al., 2010). The sequences of all primers used for qPCR analysis are described in Supplementary Table S2.

\section{Ethylene and Pollination Treatment}

Petunia flowers were treated with ethylene according to the previously described protocols (Yang et al., 2015). Petunia flowers were harvested at anthesis and their stems re-cut to $5 \mathrm{~cm}$, placed in flasks with distilled water, and subsequently treated with $2 \mu \mathrm{l}$ $1^{-1}$ ethylene for $0,2,4,8,12$, and $24 \mathrm{~h}$. To measure the expression of PhEIL1 and PhEIL2 after pollination, three flowers from each of six plants (18 flowers in total per genotype) for different lines were self-pollinated on the plant on the day before anthesis (Shibuya et al., 2004). Corollas from 8 to 10 flowers were collected at each time point, immediately frozen in liquid nitrogen, and stored at $-80^{\circ} \mathrm{C}$ for later RNA extraction.

\section{Agroinoculation of TRV Vectors}

Approximately 250 bp $3^{\prime}$ untranslated regions of PhEIL1 and PhEIL2 were cloned into the pTRV2-CHS vector to formed TRV2-CHS-PhEIL1 and TRV2-CHS-PhEIL2 vectors using their respective forward and reverse primers (Supplementary Table S3). pTRV1 and different pTRV2 derivatives vectors were transformed into Agrobacterium tumefaciens (strain GV3101) (Spitzer-Rimon et al., 2010). The Agrobacterium culture was grown overnight at $28^{\circ} \mathrm{C}$ in Luria-Bertani medium with $50 \mathrm{mg}$ $1^{-1}$ kanamycin and $200 \mathrm{mM}$ acetosyringone. The cells were harvested and resuspended in inoculation buffer containing $10 \mathrm{mM}$ MES, pH 5.5, $200 \mathrm{mM}$ acetosyringone, and $10 \mathrm{mM} \mathrm{MgCl}_{2}$ to an $\mathrm{OD}_{600}$ of 10 . Following an additional $3 \mathrm{~h}$ of incubation at $28^{\circ} \mathrm{C}$, the bacteria containing pTRV1 were mixed with the bacteria containing the pTRV2 derivatives in a 1:1 ratio. Next, 200 to $300 \mathrm{ml}$ of this mixture was applied to the cut surface of petunia plantlets after the removal of the apical meristems.

\section{Flower Longevity}

Flower longevity was measured according to previously described methods (Tan et al., 2014). To measure flower senescence, three flowers were removed from each of 20 plants (60 flowers in total per genotype) from the wild type (purple) and VIGS-mediated gene suppression lines (white) on the day before anthesis, and the flowers were placed in vials of water. The flowers were then placed in a growth room under continuous fluorescent light at $24-26^{\circ} \mathrm{C}$, and the day on which each flower completely wilted was recorded.

The data were analyzed using the ANOVA function of SAS 8.02 (Cary, NC, USA) to compare differences among genotypes. Tukey's honestly significant difference mean-separation test was used to calculate the mean separation at the $0.05 \%$ level (HSD0.05).

\section{Deletion Mutant Construction of PhEIL2 and Analyze of Transactivation Activity}

The deletion mutants of PhEIL2 were constructed by PCR. The sequences of all primers used for various PhEIL2 deletion mutants are described in Supplementary Table S4. The PCR products were fused in frame to the yeast GAL4 DNA-binding domain expression vector pGBKT7. The constructed vectors were transformed into Saccharomyces cerevisiae strain Y2HGold (Clontech, Palo Alto, CA, USA). The $\beta$-galactosidase assay was performed according to the kit instructions (Clontech).

\section{Yeast Two-Hybrid Analysis}

The coding sequence of PhEIL2 was cloned into the bait vector pGBKT7, and the coding sequences of PhEIL1 were cloned into the prey vector pGADT7. The gene-specific primers of the three genes are shown in Supplementary Table S5. The mating reactions were performed between the two haploid strains containing the pGBKT7-PhEIL2 and pGADT7PhEIL1 constructs and were plated on double dropout medium (DDO medium, SD/-Leu/-Trp) (BD Biosciences Clontech). The transformants were further streaked on quadruple dropout medium (QDO medium, SD/-Trp/-Leu/-His/-Ade) and were confirmed with a color change on $\beta$-galactosidase filter paper using a flash-freezing filter assay (Tan et al., 2014).

\section{Bimolecular Fluorescence Complementation (BiFC) Assay}

The full-length PhEIL1 and PhEIL2 cDNAs were inserted into pSAT-1628 (pYFC) and pSAT-1882 (pYFN) to form the pYFCPhEIL1 and pYFN-PhEIL2 vectors, respectively. The sequences of all primers used for BiFC are described in Supplementary Table S6. pYFC-PhEIL1 and pYFN-PhEIL2, pYFC-PhEIL1 and pSATYFC, pSAT-YFN, and pYFN-PhEIL2 were co-transformed into petunia protoplasts with the pSAT-GFP as positive control (Tan et al., 2014).

Leaves of 5- to 6-week-old petunia plants were used for the preparation of protoplasts. The vectors were used in polyethylene glycol-mediated transformation of the petunia protoplasts (Spitzer-Rimon et al., 2010). The protoplasts were assayed for fluorescence $12-24 \mathrm{~h}$ after transformation. The images were produced by the confocal laser scanning system (ECLIPSE TE2000-E; Nikon, Tokyo, Japan).

\section{Statistical Analyses}

Statistical analysis was performed using one way analysis of variance (ANOVA) followed by Duncan's multiple range test (DMRT) with three replicates. $P$-values $\leq 0.05$ were considered as significant.

\section{RESULTS}

\section{Identification of a PhEIL Transcription Factor Gene in Petunia}

A PhEIL transcription factor gene full-length cDNA was isolated in the petunia 'Ultra', named PhEIL2, since PhEIL1 had been previously submitted to GeneBank. PhEIL2 was predicted to encode a 612 amino acid protein, with a calculated molecular weight of $69.1 \mathrm{kDa}$. The multiple sequence alignments of EILlike proteins in petunia, tomato and Arabidopsis are presented in Supplementary Figure S1. PhEIL1 shares 35.1, 35.6, 29.7, 50.1, and $34.6 \%$ amino acid sequence identity with PhEIL2, AtEIL1, 
AtEIL2, AtEIL3, and AtEIN3. PhEIL2 shares 62.7, 40.2, 39.3, and $61.8 \%$ amino acid sequence identity with AtEIL1, AtEIL2, AtEIL3, and AtEIN3 (Supplementary Table S1). The N-terminal half of the deduced protein of PhEIL1 and PhEIL2 had higher similarity to the corresponding regions of AtEIL3 and AtEIL1 in Arabidopsis than their C-terminal half to the corresponding regions of the latter two, respectively (Supplementary Figure S1). As found in other EIN3 homologs, the PhEIL1 and PhEIL2 proteins possess an amino-terminal acidic region, five small clusters of basic amino acids regions and a Pro-rich domain (158-198; 201-242, respectively) (Supplementary Figure S1). The acidic and Pro-rich regions have been proposed to be functional as transcriptional activation domains (Chao et al., 1997). Acidic and proline-rich regions have been widely described as transcriptional activation domains (Mitchell and Tjian, 1989) and may serve such a role in the EIN3/EIL proteins. PhEIL1 and PhEIL2 have a Lys residue at positions 203 and 247, respectively, which is required for the function of EIN3 (Chao et al., 1997; Solano et al., 1998; Alonso et al., 2003). In addition, phylogenetic tree showed that PhEIL1 is the homologs of AtEIL3 and PhEIL2 is the homologs of AtEIL1 and AtEIN3 (Supplementary Figure S2).

\section{Expression of PhEIL1 and PhEIL2}

Quantitative real-time PCR (qPCR) analysis showed that both PhEIL1 and PhEIL2 mRNA levels are high in corollas and ovaries, and the expression PhEIL1 is the lowest in stems, while that of PhEIL2 is the lowest in leaves (Figure 1A). During natural flower senescence, the PhEIL1 mRNA level did not show significant change before day 3 but increased rapidly from days 4 to 6 . The expression of PhEIL2 decreased before day 3, but then increased until day 7 (Figure 1B). After ethylene treatment, PhEIL1 mRNA levels significantly increased from hours 2 to 24, whereas PhEIL2 expression decreased from 0 to $8 \mathrm{~h}$ and then kept stable level until to $24 \mathrm{~h}$ (Figure 1C). In petunia, pollination induced an ethylene burst and consequently floral senescence (Shibuya et al., 2004). As shown as in Figure 1D, the mRNA levels of PhEIL1/PhEIL2 were significantly increased after pollination $8 \mathrm{~h}$ by qPCR analysis.

\section{Silencing of Both PhEIL1 and PhEIL2 Delays Flower Senescence and Decrease Ethylene Production}

The VIGS system with PhCHS as the reporter gene has been established in the petunia 'Ultra' (Violet line) by us (Tan et al.,
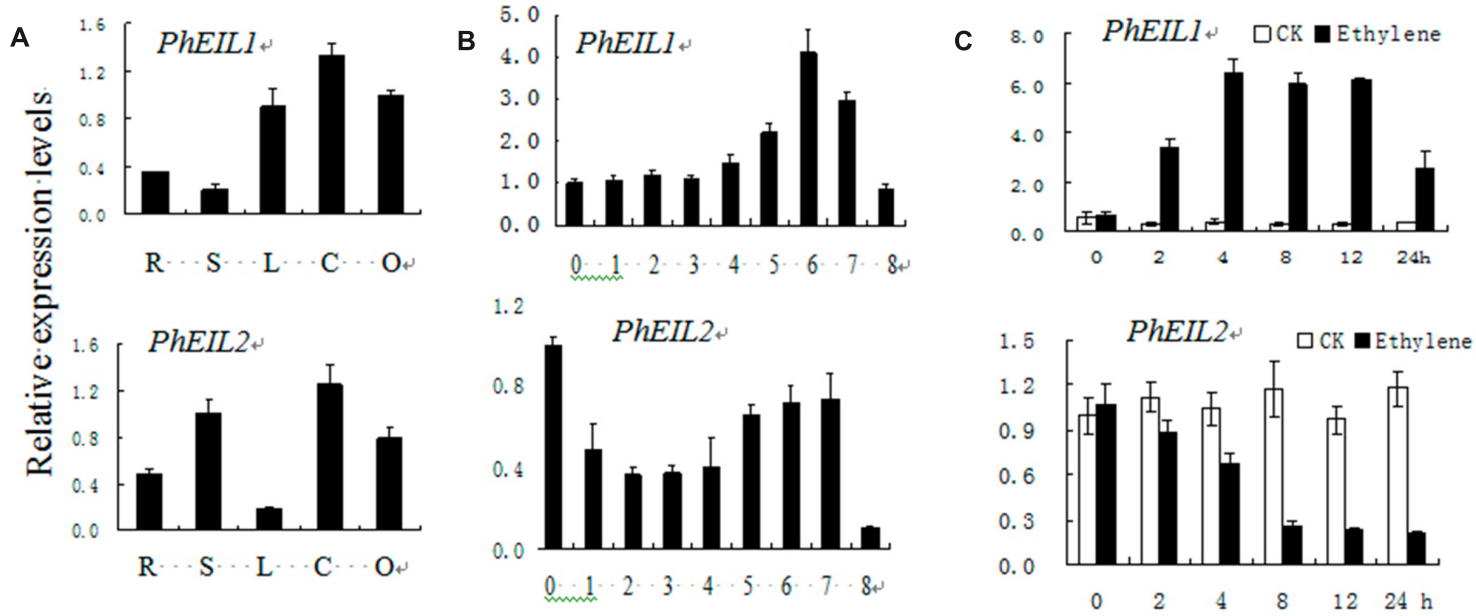

D
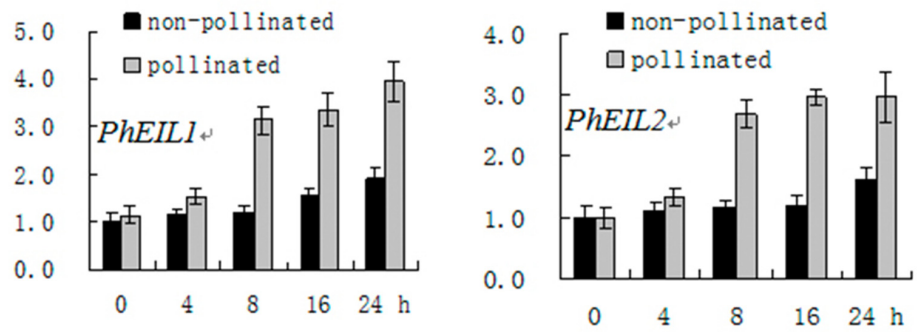

FIGURE 1 | Temporal and spatial expression analysis of PhEIL1 and PhEIL2 by quantitative real-time PCR. Expression analysis of PhEIL1 and PhEIL2 in different organs (A), in corollas during natural flower senescence (B), response to ethylene (C), and pollination (D). R, roots; L, leaves; S, stems; C, corollas, O, ovaries. Relative expression levels are shown as fold change values. Data are the mean $\pm \operatorname{SD}(n=3)$. Statistical analysis was performed using one way analysis of variance (ANOVA) followed by Duncan's multiple range test (DMRT) with three replicates. $P$-values $\leq 0.05$ were considered as significant. 

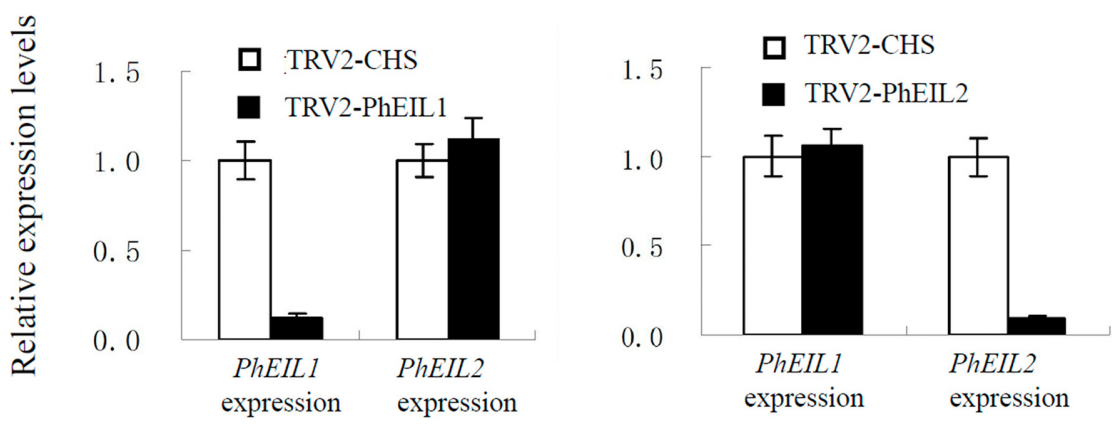

FIGURE 2 | Effects of TRV2-CHS/PhEIL1 and TRV2-CHS/PhPhEIL2 treatment on the expression of PhEIL1 and PhEIL2 in white flowers on day 4 after opening by quantitative real-time PCR, respectively. Relative expression levels are shown as fold change values. Data are mean \pm SD $(n=3)$. Statistical analysis was performed using one way analysis of variance (ANOVA) followed by Duncan's multiple range test (DMRT) with three replicates. $P$-values $\leq 0.05$ were considered as significant.

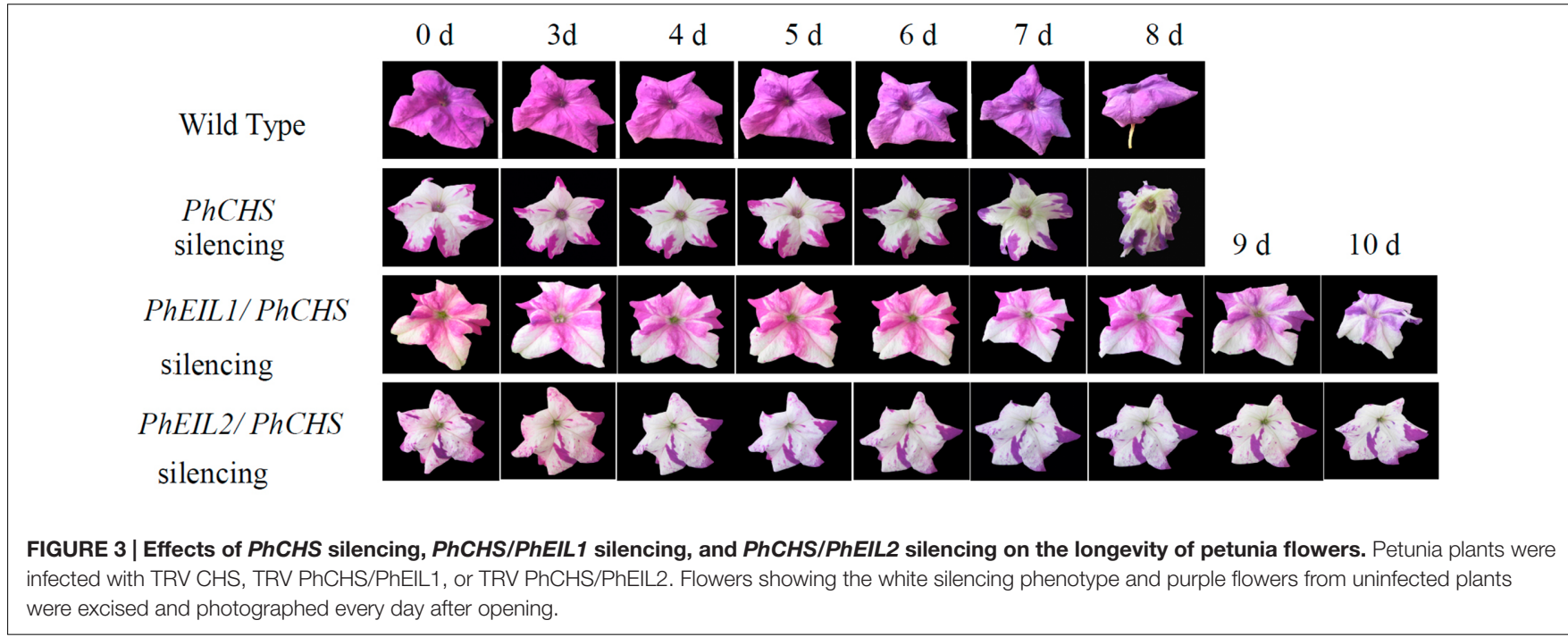

2014). To identify the effects of PhEIL1 and PhEIL2 silencing on flower senescence, TRV-CHS-PhEIL1 and TRV-CHS-PhEIL2 vectors, which were inserted approximately $250 \mathrm{bp}$ fragments of $3^{\prime}$ untranslated sequences of the petunia PhEIL1 and PhEIL2 cDNAs into a pTRV2-CHS vector, respectively, were constructed. The PhEIL1 and PhEIL2 mRNA levels in the white flowers in the TRV-CHS-PhEIL1 and TRV-CHS-EIL2 treatments, respectively, decreased to less than 20\% relative to control (TRV-CHS), and PhEIL2 and PhEIL1 mRNA levels did not significantly changed in the flowers of PhEIL1 and PhEIL2 silencing, respectively (Figure 2).

As shown in Figure 3 and Table 1, the longevity of the flowers of PhCHS/PhEIL1 silencing and PhCHS/PhEIL2 silencing was increased compared with that of the flowers from plants of PhCHS silencing and wild type plants.

The effects of PhEIL1 and PhEIL2 silencing on ethylene production were examined. As shown in Figure 4, white flowers of both PhCHS/PhEIL1 and PhCHS/PhEIL2 silencing produced less ethylene than those of $\mathrm{PhCHS}$ silencing (TRV-CHS treatment) in days 4 and 5 after anthesis.

\section{Silencing of Both PhEIL1 and PhEIL2 Reduces the Expression of PhERF3 and PhCP2}

In petunia, PhERF3 (HQ259597) and PhCP2 (AY662988) are regard as senescence-associated genes (Jones et al., 2005; Liu et al., 2010). To further examine the involvement of PhEIL1 and PhEIL2 in flower senescence, the expression of PhERF3 and PhCP2 was examined by qPCR in PhEIL1- and PhEIL2-silenced flowers. Expression of PhERF3 and PhCP2 in white flowers with PhCHS/PhEIL1 and PhCHS/PhEIL2 silencing was significantly lower than that with $P h C H S$ silencing after the flowers were open for 4 and 5 days (Figure 5).

\section{The PhElL2 Protein Activate Transcription in Yeast}

Previous research suggested that the $\mathrm{N}$-terminal acidic region (1-50 amino acids) of mung bean (Vigna radiata) VR-EIL2, a transcriptional activator, is the transcriptional domain (Lee and Kim, 2003). In order to determine the transcriptional domain, PhEIL2 and its several deletion mutant vectors were constructed 
TABLE 1 | The effects of PhEIL1 and PhEIL2 suppression on the longevity of petunia flowers.

\begin{tabular}{lllll}
\hline & Wild type & $\begin{array}{l}\text { PhCHS } \\
\text { suppression }\end{array}$ & $\begin{array}{l}\text { PhEIL1 } \\
\text { suppression }\end{array}$ & $\begin{array}{l}\text { PhEIL2 } \\
\text { suppression }\end{array}$ \\
\hline $\begin{array}{l}\text { longevity of } \\
\text { flowers (day) }\end{array}$ & $6.96 \pm 0.82^{\mathrm{c}}$ & $7.0 \pm 0.8^{\mathrm{c}}$ & $8.4 \pm 0.9^{\mathrm{b}}$ & $10.6 \pm 1.3^{\mathrm{a}}$ \\
\hline
\end{tabular}

Data were expressed as mean $\pm S D$. Different letters indicate significant difference among treatments at the 0.05 significance level based on Duncan's multiple-range test. Error bars indicate standard error of the mean $(n=3)$.

and the behavior of each construct was investigated in yeast cells. The full-length PhEIL2 protein fused to the GAL4 DNA-binding domain effectively activates the expression of the reporter gene of lacZ (Figures 6A,B), which indicates the function of PhEIL2 as a transcriptional activator in yeast. Further transcriptional activity analysis of various deletion mutants of PhEIL2 showed that the transcription-stimulating activity was still apparent when the C-terminal regions (353-470 and 471-612 amino acids) were fused to the GAL4 DNA-binding domain; both PhEIL2 $353-470$ and PhEIL2 $471-612$ mutant protein contained $\beta$-galactosidase activity while the PhEIL $2_{1-352}$ mutant protein did not (Figure 6). Thus, the 353-612-amino acids at C-terminal of PhEIL2 play an important role for the function of PhEIL2 as a transcriptional activator.

\section{PhEIL2 Interacts with PhEIL1 by Y2H and BiFC Assays}

Since EILs could interact with its target as dimmers (Solano et al., 1998) and both PhEIL1 and PhEIL2 are involved in flower senescence in petunia, it is necessary to test whether PhEIL1 interacts with PhEIL2 in petunia and yeast two-hybrid

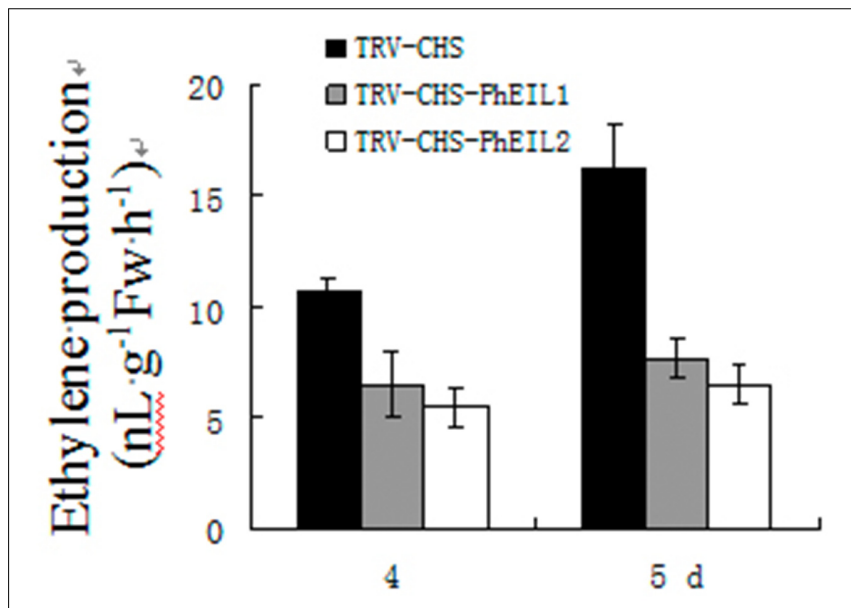

FIGURE 4 | Ethylene productions in PhEIL1 and PhEIL2 silencing flowers. Ethylene production was measured for flower corollas after the flowers were open for 4 and 5 days. Ethylene was collected for $2 \mathrm{~h}$ and subsequently measured using a gas chromatograph. Ethylene production rates were calculated based on tissue FW. Mean \pm SE values were determined from five samples. Statistical analysis was performed using one way analysis of variance (ANOVA) followed by Duncan's multiple range test (DMRT) with three replicates.
$(\mathrm{Y} 2 \mathrm{H})$ assay was performed. To avoid this activation of the reporter gene, the $5^{\prime}$ ORF of PhEIL 2 cDNA sequence, encoding $\mathrm{N}$-terminal half of the deduced protein (amino acids 1-352), and the full length cDNA of PhEIL1 were subcloned into the pGBKT7 and pGADT7 vectors to form pGBKT7-PhEIL2 $2_{1-352}$ bait vector and pGADT7-PhEIL1 prey vector, respectively. The derivative vectors of pGBKT7 and the pGADT7 were co-transformed into the yeast strain Y187. Yeast cells co-transformed with pGBKT7- PhEIL2 1-352+pGADT7-PhEIL1 grew on selective medium lacking Trp, Leu, His, and Ade in the presence of $5 \mathrm{mM}$ 3-AT. On the contrary, yeast cells harboring pGBKT7+pGADT7PhEIL1, or pGADT7-PhEIL2 $1-352+$ pGBKT7 could not grow on the same selective medium (Figures 7A,B). These data showed that PhEIL2 $2_{1-352}$ interacts with PhEIL1 in yeast.

To further test the interaction between PhEIL2 and PhEIL1, BiFC assay was performed. Co-expression of the C-terminal half of YFP fused to PhEIL1 (cYFP-PhEIL1) and the N-terminal half of YFP fused to PhEIL2 (nYFP-PhEIL2) in petunia protoplasts led to fluorescence with GFP as positive control. No interaction was detected between nYFP-PhEIL2 and cYFP, or between nYFP and cYFP-PhEIL1, which confirms the interaction between PhEIL2 and PhEIL1 (Figures 7C-F). In addition, previous study showed that EOBII was located in nuclear (Spitzer-Rimon et al., 2010) and with RFP (Red Fluorescent Protein)-EOBII as the nuclear maker, the interaction between PhEIL2 and PhEIL1 was further confirmed (Figure 7G).

\section{DISCUSSION}

Ethylene responsiveness in petunia corollas increases highly during flower senescence (Shibuya et al., 2004). In this study, a full-length cDNA of petunia EIL gene, PhEIL2, was isolated and the characters of PhEIL2 and PhEIL2 were identified during flower senescence.

When compared with other EILs described to date from different organisms, the N-terminal half of the deduced protein of PhEIL1 and PhEIL2 had high similarity to the corresponding regions of AtEIL3 and AtEIL1 in Arabidopsis, suggesting that they are indeed functional EIN3-like genes (Chao et al., 1997).

Previous research showed that other organisms EILs expression are regulated in tissue-specific manners (Waki et al., 2001; Lee and Kim, 2003; Iordachescu and Verlinden, 2005). Similarly, in this study, the relative expression pattern of the two different mRNAs varied in these tissues, with both PhEIL1 and PhEIL2 transcript being predominantly present in corollas and up-regulated during flower senescence.

The expression of most of EILs of other organisms, such as Arabidopsis EIN3, tomato LeEILs, and mung bean $V r-E I L 1$ and $V r$-EIL2, was not significantly changed by the treatment of exogenous ethylene in grown plants (Chao et al., 1997; Lee and Kim, 2003). However, in this study, PhEIL1 mRNA was up-regulated by ethylene, which is in line with the result of previous report (Shibuya et al., 2004), while PhEIL2 transcriptional level is down-regulated by ethylene. Similarly, DC-EIL3 mRNA showed significant accumulation while DC-EIL1 mRNA showed significant reduction upon ethylene exposure in 

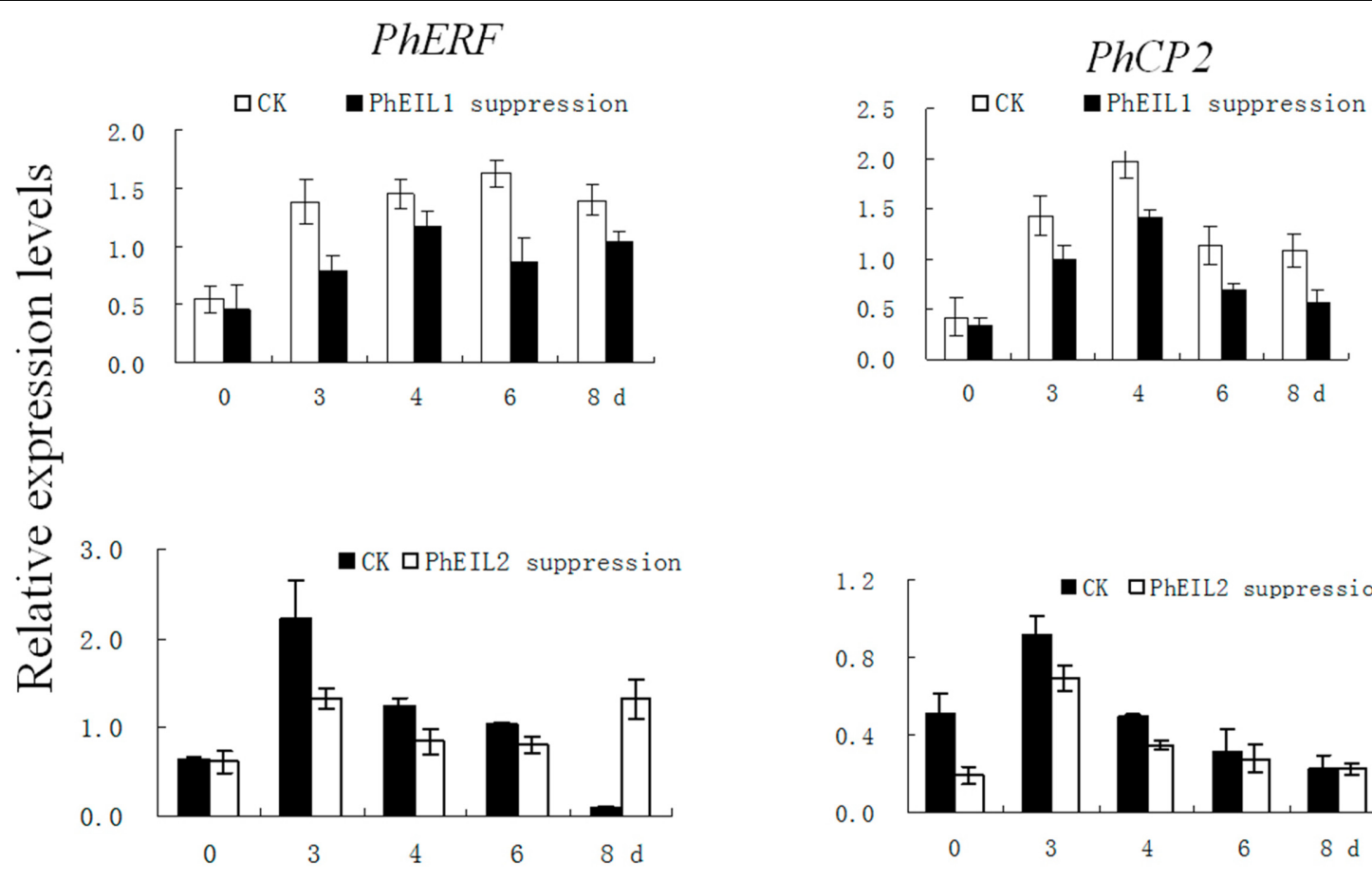

FIGURE 5 | Effects of PhEIL1 and PhEIL2 silencing on the expression of PhERF3 and PhCP2 in flowers. Flowers were detached on the day in anthesis. Total RNA was isolated from white flower tissues. PhERF3 and PhCP2 mRNA levels were determined by quantitative real-time PCR. Relative expression levels are shown as fold change values. Data are the mean $\pm \operatorname{SD}(n=3)$. Statistical analysis was performed using one way analysis of variance (ANOVA) followed by Duncan's multiple range test (DMRT) with three replicates. $P$-values $\leq 0.05$ were considered as significant.

A

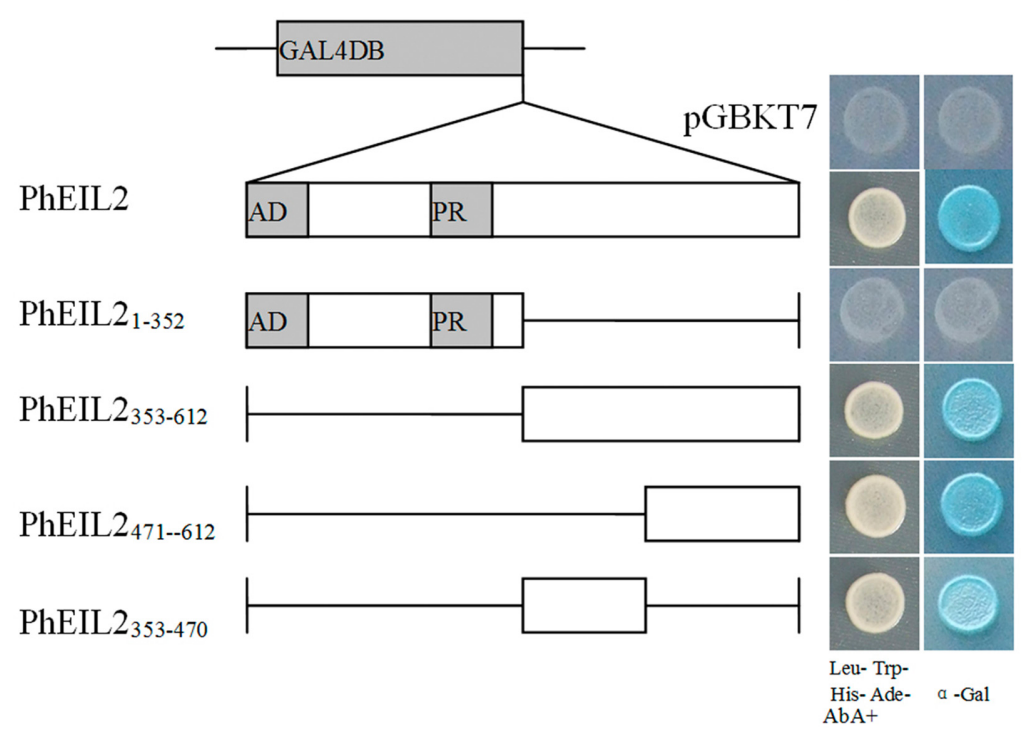

FIGURE 6 | GAL4 DB-PhEIL2 fusions and their effect on transcriptional activation of the lac Z reporter gene in yeast cells. (A) Schematic overview of the fusion proteins between the GAL4 DNA-binding domain (DB) and various deletion mutants of PhEIL2 that were investigated for transcription-stimulating activity in yeast. (B) The PhEIL2 and its deletion constructs fused in the GAL4 DB expression vector were transformed into yeast strain Y2HGold. The transformants were selected by growth on Trp- and Leu- medium at $30^{\circ} \mathrm{C}$ for 3 days. Yeast transformants were tested for growth in the absence of His, Trp, Leu, and Ade but containing $125 \mu \mathrm{M}$ Aureobasidin A as well as turn blue in the presence of the chromagenic substrate X- $\beta$-Gal was scored as a positive interaction. The yeast strain is carrying a modified HIS gene whose transcription is under the control of GAL4 operator. 


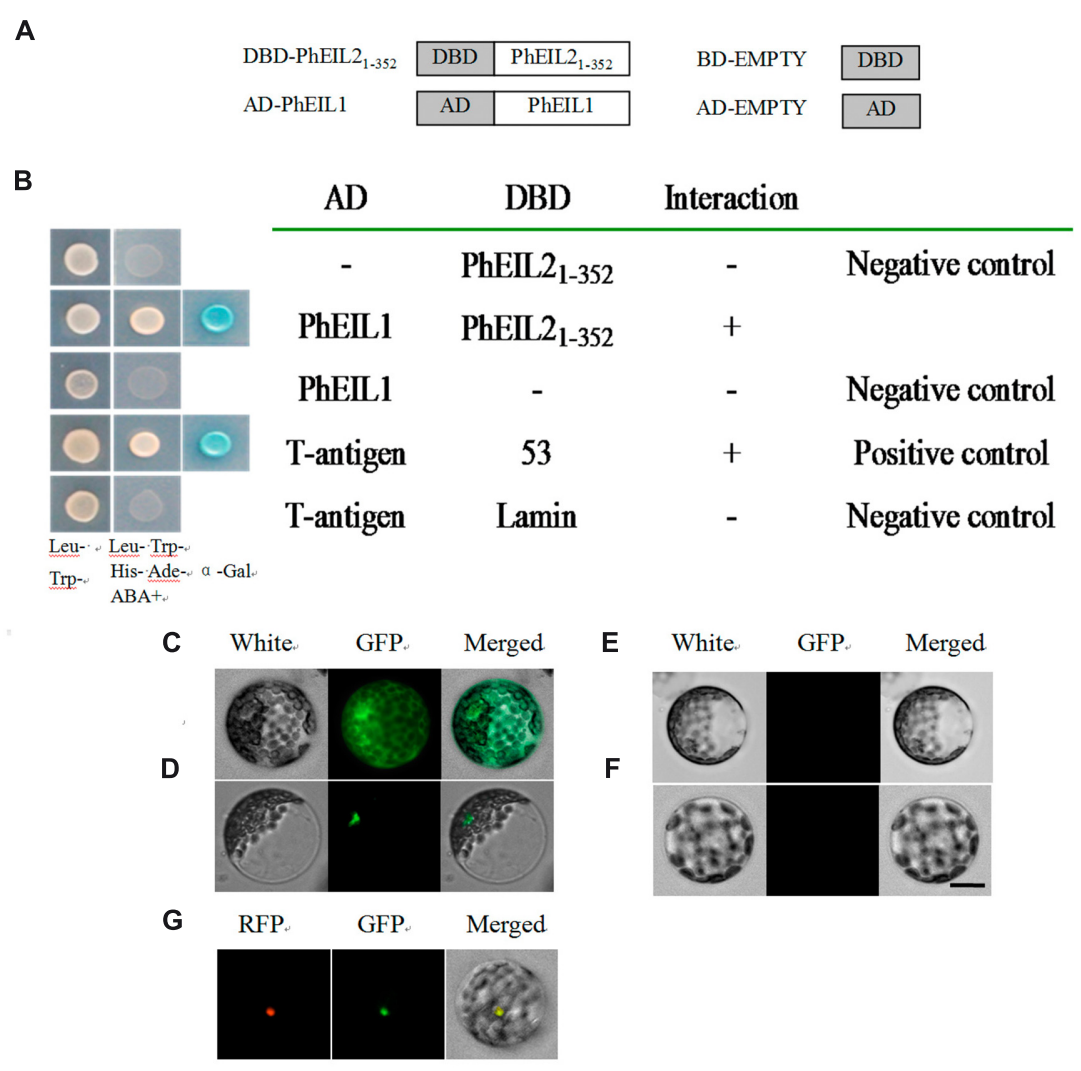

FIGURE 7 | Interaction between PhEIL2 $\mathbf{1 - 3 5 2}$ and portions of PhEIL1. (A,B) Yeast two-hybrid assays between PhEIL2 1 - 352 and portions of PhEIL1. (A) Portions of PhEIL2 and PhEIL1 were fused to DBD and AD, respectively; (B) interaction between PhEIL2 $1-352$ and PhEIL1. Gold Y2H yeast strains were co-transformed with DBD-PhEIL2 $1-352$ and PhEIL1. The ability of yeast cells to grow on synthetic medium lacking tryptophan, leucine, histidine, and adenine but containing $125 \mu \mathrm{M}$ Aureobasidin A as well as turn blue in the presence of the chromagenic substrate X- $\beta$-Gal was scored as a positive interaction. Yeast cells transformed with pGBKT7-53+pGADT7-T, pGBKT7-PhEIL2 1-352+pGADT7-T, pGBKT7-PhEIL2 1-352 +pGADT7-PhEIL1, pGBKT7+pGADT7-PhEIL1, or pGBKT7-Lamin+pGADT7-T were included as positive or negative controls. C-F, BiFC assays between PhEIL2 and PhEIL1. Translational fusion constructs of the coding region of PhEIL2 to PSAT-YFC and the coding region of PhEIL1 to pSAT-YFN were transferred into petunia protoplasts and tested for fluorescence complementation. (C) pSAT-GFP; (D) YFC-PhEIL2 and YFN-PhEIL1; (E) YFC-PhEIL2 and pSAT-YFN; (F) pSAT-YFC and YFN-PhEIL1. (G) YFC-PhEIL2, YFN-PhEIL1, and RFP-EOBII. Three biological replicates do these results represent in (G). Images of (C-F) were captured with a confocal laser scanning system. Scale bars: $5 \mu \mathrm{m}$.

carnation (Dianthus caryophyllus) (Waki et al., 2001; Iordachescu and Verlinden, 2005). It is possible that the activities of EILs are controlled by a posttranslational mechanism (Chao et al., 1997; Kosugi and Ohashi, 2000; Tieman et al., 2001; Lee and Kim, 2003). Moreover, EIN3 and EIL1 proteins are degraded by EBF1/2 in ethylene signaling (Potuschak et al., 2003).

In petunia, pollination induced an ethylene burst and consequently floral senescence (Shibuya et al., 2004). The mRNA levels of both PhEIL1 and PhEIL2 were significantly increased after pollination 8 h by qPCR analysis, although PhEIL2 expression showed down-regulated by exogenous ethylene treatment, suggesting that PhEIL2 expression is regulated by multi-factors after pollination.

The etiolated T2 generation seedlings of Arabidopsis plants overexpressing the EIN3 and EIL1 genes and TEIL CDNA, showed a phenotype of constitutive triple response under the condition of lack of exogenous ethylene (Chao et al., 1997; Kosugi and
Ohashi, 2000). Consistent with these results, VIGS-mediated both PhEIL1 and PhEIL2 silencing delayed flower senescence in petunia in this study. Furthermore, the expression of two senescence-associated genes, PhERF3 and PhCP2, decreased in flowers in which VIGS-mediated silencing of PhEIL1 and PhEIL2 occurred. In addition, both PhCHS/PhEIL1 and PhCHS/PhEIL2 silencing led less ethylene production than $P h C H S$ suppression in white flowers in days 4 and 5 after anthesis, which may suggest it exerts feedback control over ethylene production and the reduced ethylene evolution could be due to the partial block of ethylene autocatalysis. These results showed that both PhEIL1 and PhEIL2 are involved in flower senescence. On the other hand, in tomato, antisense plants with reduced transcription of a single LeEIL did not result in notable changes in ethylene response, but reduced the mRNA levels of multiple LeEIL reduced significantly ethylene response, showing functional redundancy of LeEILs in tomato (Tieman et al., 2001). 


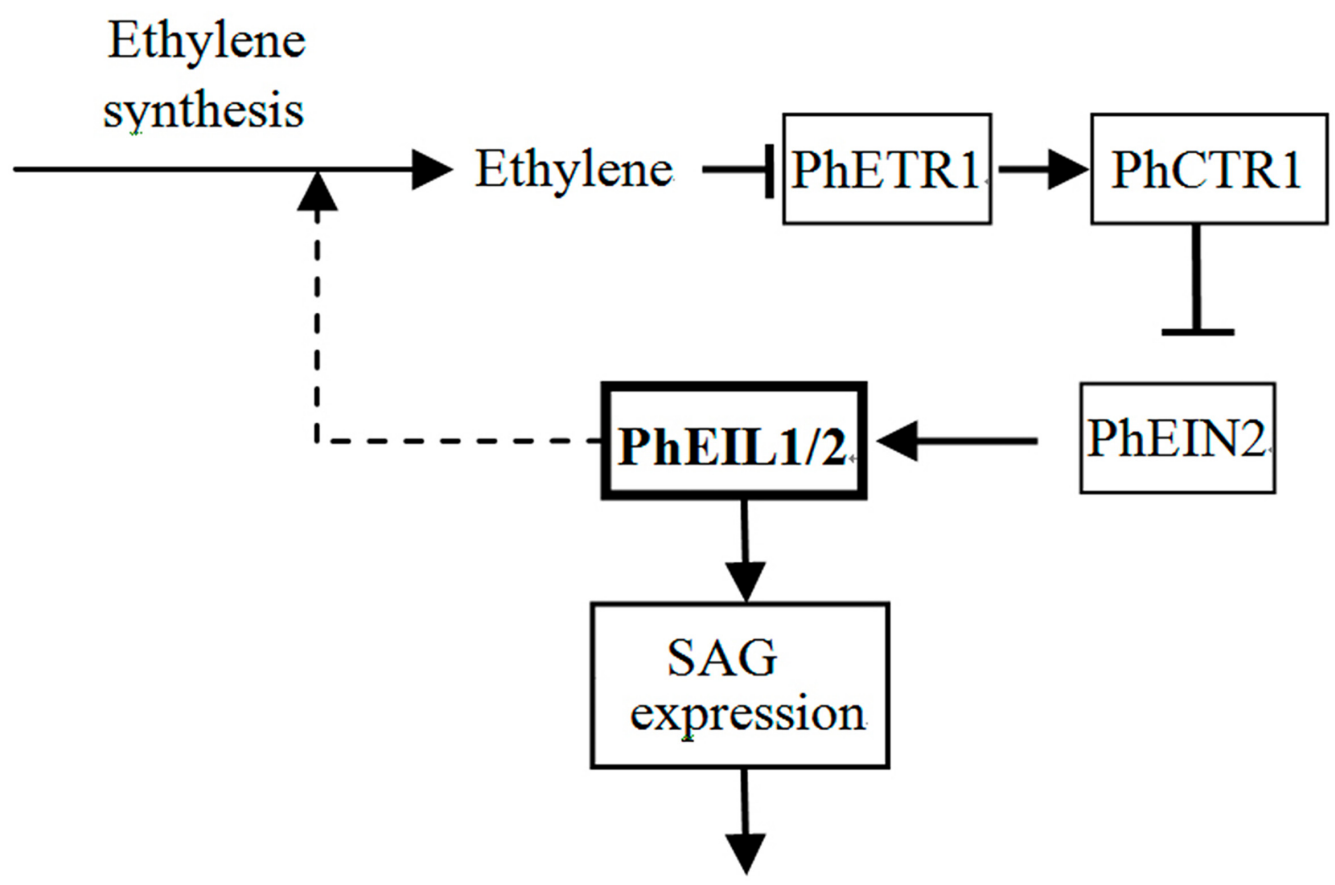

Flower:Senescence

FIGURE 8 | Proposed models for the involvement of PhEIL1 and PhEIL2 in flower senescence in petunia.

To uncover the transcriptional activation domain of PhEIL2, yeast one-hybrid assay was performed using several PhEIL2 deletion mutants. The results showed that the C-terminal 353612 amino acid region that consists of 260 amino acid residues is the essential domain for transcription-stimulating activity. In contrast, the essential domain for transcription-stimulating activity in mung bean VR-EIL2 was laid in the acidic region that comprises 50 amino acid residues in N-terminal. In our experimental conditions, the PhEIL $2_{1-353}$ mutant protein has only the background level of activity in yeast, suggesting that it is not essential domain for transcriptional activation. Further analysis showed that acid domain of PhEIL2 shares only 58.3\% identity with that of Vr-EIL2. These results showed that essential domain for transcription-stimulating activity is not conserved in different EILs or in different species and requires further study.

Previous study suggested that homodimers of both EIN3 and EIL1 proteins are able to bind the promoters of ERF1, while EIN3 and EILs are not capable of forming heterodimers in Arabidopsis (Solano et al., 1998). In contrast, in this study, PhEIL2 interacts with PhEIL1 by $\mathrm{Y} 2 \mathrm{H}$ and BiFC assays. In addition, both PhEIL1 and PhEIL2 are involved senescence. So, it is possible that heterodimers of PhEIL1 and PhEIL2 recognize their targets in vivo.

Based on the experimental data presented here and the model of ethylene signaling in Arabidopsis (Ji and Guo, 2013), we proposed a model to explain the involvement of the PhEIL1 and PhEIL2 in senescence in petunia flowers (Figure 8). In the present of ethylene, ethylene binds the receptor PhETR1 and ethylene signaling is transmitted to PhEIL1 and PhEIL2 heterodimers through PhCTR1 and PhEIN2 in petunia flowers (Shibuya et al., 2004). The heterodimers then activate the expression of senescence-related genes and accelerate senescence of flowers. In contrast, PhEIL1 or PhEIL2 suppression leads to the reduction of the heterodimers and the expression of senescence-related genes, and delays senescence of flowers. At the same time, ethylene production is reduced in petunia corollas, suggesting that PhEIL1 and PhEIL2 heterodimers could be involved in the regulation of the biosynthesis of ethylene in petunia flowers.

\section{AUTHOR CONTRIBUTIONS}

YY and JL designed research. FL, LH, YC, HL, and JL performed research. YY and FL, wrote paper.

\section{FUNDING}

This work was supported by National Natural Science Foundation of China (31270736, 31170653, and 31470700).

\section{SUPPLEMENTARY MATERIAL}

The Supplementary Material for this article can be found online at: http://journal.frontiersin.org/article/10.3389/fpls.2016.01606/ full\#supplementary-material 


\section{REFERENCES}

Abeles, F. B., Morgan, P. W., Saltveit, M. E. Jr., Abeles, F. B., Morgan, P. W., and Saltveit, M. E. Jr. (1992). Regulation of ethylene production by internal, environmental and stress factors. Ethylene Plant Biol. 2, 56-119. doi: 10.1016/B978-0-08-091628-6.50010-2

Alonso, J. M., Hirayama, T., Roman, G., Nourizadeh, S., and Ecker, J. R. (1999). EIN2, a bifunctional transducer of ethylene and stress responses in Arabidopsis. Science 284, 2148-2152. doi: 10.1126/science.284.5423.2148

Alonso, J. M., Stepanova, A. N., Leisse, T. J., Kim, C. J., Chen, H., Shinn, P., et al. (2003). Genome-wide insertional mutagenesis of Arabidopsis thaliana. Science 301, 653-657. doi: 10.1126/science.1086391

Borochov, A., and Woodson, W. R. (1989). Physiology and biochemistry of flower petal senescence. Hortic. Rev. 11, 15-43. doi: 10.1002/9781118060841.ch2

Chang, C., Kwok, S. F., Bleecker, A. B., and Meyerowitz, E. M. (1993). Arabidopsis ethylene-response gene ETR1: similarity of product to twocomponent regulators. Science 262, 539-544. doi: 10.1126/science.8211181

Chao, Q., Rothenberg, M., Solano, R., Roman, G., Terzaghi, W., and Ecker, J. R. (1997). Activation of the ethylene gas response pathway in Arabidopsis by the nuclear protein ETHYLENE-INSENSITIVE3 and related proteins. Cell 89, 1133-1144. doi: 10.1016/S0092-8674(00)80300-1

Gagne, J. M., Smalle, J., Gingerich, D. J., Walker, J. M., Yoo, S., Yanagisawa, S., et al. (2004). Arabidopsis EIN3-binding F-box 1 and 2 form ubiquitinprotein ligases that repress ethylene action and promote growth by directing EIN3 degradation. Proc. Natl. Acad. Sci. U.S.A. 101, 6803-6808. doi: 10.1073/pnas.0401698101

Guo, H., and Ecker, J. R. (2003). Plant responses to ethylene gas are mediated by SCF EBF1/EBF2-dependent proteolysis of EIN3 transcription factor. Cell 115, 667-677. doi: 10.1016/S0092-8674(03)00969-3

Hua, J., Chang, C., Sun, Q., and Meyerowitz, E. M. (1995). Ethylene insensitivity conferred by Arabidopsis ERS gene. Science 269, 1712-1714. doi: $10.1126 /$ science. 7569898

Hua, J., Sakai, H., Nourizadeh, S., Chen, Q. G., Bleecker, A. B., Ecker, J. R., et al. (1998). EIN4 and ERS2 are members of the putative ethylene receptor gene family in Arabidopsis. Plant Cell 10, 1321-1332. doi: 10.1105/tpc.10.8.1321

Iordachescu, M., and Verlinden, S. (2005). Transcriptional regulation of three EIN3-like genes of carnation (Dianthus caryophyllus L. cv. Improved White Sim) during flower development and upon wounding, pollination, and ethylene exposure. J. Exp. Bot. 56, 2011-2018. doi: 10.1093/jxb/eri199

Ji, Y., and Guo, H. (2013). From endoplasmic reticulum (ER) to nucleus: EIN2 bridges the gap in ethylene signaling. Mol. Plant 6, 11-14. doi: $10.1093 / \mathrm{mp} / \mathrm{sss} 150$

Jones, M. L., Chaffin, G. S., Eason, J. R., and Clark, D. G. (2005). Ethylenesensitivity regulates proteolytic activity and cysteine protease gene expression in petunia corollas. J. Exp. Bot. 56, 2733-2744. doi: 10.1093/jxb/eri266

Ju, C., Yoon, G. M., Shemansky, J. M., Lin, D. Y., Ying, Z. I., Chang, J., et al. (2012). CTR1 phosphorylates the central regulator EIN2 to control ethylene hormone signaling from the ER membrane to the nucleus in Arabidopsis. Proc. Natl. Acad. Sci. U.S.A. 109, 19486-19491. doi: 10.1073/pnas.1214848109

Kieber, J. J., Rothenberg, M., Roman, G., Feldmann, K. A., and Ecker, J. R. (1993). CTR1, a negative regulator of the ethylene response pathway in Arabidopsis, encodes a member of the raf family of protein kinases. Cell 72, 427-441. doi: 10.1016/0092-8674(93)90119-B

Kosugi, S., and Ohashi, Y. (2000). Cloning and DNA-binding properties of a tobacco Ethylene-Insensitive3 (EIN3) homolog. Nucleic Acids Res. 28, 960-967. doi: $10.1093 /$ nar/28.4.960

Lee, J., and Kim, W. T. (2003). Molecular and biochemical characterization of VREILs encoding mung bean ETHYLENE INSENSITIVE3-LIKE proteins. Plant Physiol. 132, 1475-1488. doi: 10.1104/pp.103.022574

Li, W., Ma, M., Feng, Y., Li, H., Wang, Y., Ma, Y., et al. (2015). EIN2-directed translational regulation of ethylene signaling in Arabidopsis. Cell 163, 670-683. doi: 10.1016/j.cell.2015.09.037

Liu, J., Li, J., Wang, H., Fu, Z., Liu, J., and Yu, Y. (2010). Identification and expression analysis of ERF transcription factor genes in petunia during flower senescence and in response to hormone treatments. J. Exp. Bot. 62, 825-40. doi: $10.1093 /$ jxb/erq324
Mallona, I., Lischewski, S., Weiss, J., Hause, B., and Egea-Cortines, M. (2010). Validation of reference genes for quantitative real-time PCR during leaf and flower development in Petunia hybrida. BMC Plant Biol. 10:4. doi: 10.1186/1471-2229-10-4

Mitchell, P. J., and Tjian, R. (1989). Transcriptional regulation in mammalian cells by sequence-specific DNA binding proteins. Science 245, 371-378. doi: 10.1126/science. 2667136

Potuschak, T., Lechner, E., Parmentier, Y., Yanagisawa, S., Grava, S., Koncz, C., et al. (2003). EIN3-dependent regulation of plant ethylene hormone signaling by two Arabidopsis F box proteins: EBF1 and EBF2. Cell 115, 679-689. doi: 10.1016/S0092-8674(03)00968-1

Qiao, H., Shen, Z., Huang, S. C., Schmitz, R. J., Urich, M. A., Briggs, S. P., et al. (2012). Processing and subcellular trafficking of ER-tethered EIN2 control response to ethylene gas. Science 338, 390-393. doi: 10.1126/science. 1225974

Schaller, G. E., and Bleecker, A. B. (1995). Ethylene-binding sites generated in yeast expressing the Arabidopsis ETR1 gene. Science 270, 1809-1811. doi: 10.1126/science.270.5243.1809

Shibuya, K., Barry, K. G., Ciardi, J. A., Loucas, H. M., Underwood, B. A., Nourizadeh, S., et al. (2004). The central role of PhEIN2 in ethylene responses throughout plant development in petunia. Plant Physiol. 136, 2900-2912. doi: 10.1104/pp.104.046979

Solano, R., Stepanova, A., Chao, Q., and Ecker, J. R. (1998). Nuclear events in ethylene signaling: a transcriptional cascade mediated by ETHYLENEINSENSITIVE3 and ETHYLENE-RESPONSE-FACTOR1. Genes Dev. 12, 3703-3714. doi: 10.1101/gad.12.23.3703

Spitzer-Rimon, B., Marhevka, E., Barkai, O., Marton, I., Edelbaum, O., Masci, T., et al. (2010). EOBII, a gene encoding a flower-specific regulator of phenylpropanoid volatiles' biosynthesis in petunia. Plant Cell 22, 1961-1976. doi: 10.1105/tpc.109.067280

Tan, Y., Liu, J., Huang, F., Zhong, S., and Yu, Y. (2014). PhGRL2 protein, interacting with PhACO1, is involved in flower senescence in the petunia. Mol. Plant 7, 1384-1387. doi: 10.1093/mp/ssu024

Tieman, D. M., Ciardi, J. A., Taylor, M. G., and Klee, H. J. (2001). Members of the tomato LeEIL (EIN3-like) gene family are functionally redundant and regulate ethylene responses throughout plant development. Plant J. 26, 47-58. doi: 10.1046/j.1365-313x.2001.01006.x

Waki, K., Shibuya, K., Yoshioka, T., Hashiba, T., and Satoh, S. (2001). Cloning of a cDNA encoding EIN3-like protein (DC-EIL1) and decrease in its mRNA level during senescence in carnation flower tissues. J. Exp. Bot. 52, 377-379. doi: 10.1093/jexbot/52.355.377

Wen, X., Zhang, C., Ji, Y., Zhao, Q., He, W., An, F., et al. (2012). Activation of ethylene signaling is mediated by nuclear translocation of the cleaved EIN2 carboxyl terminus. Cell Res. 22, 1613. doi: 10.1038/cr.2012.145

Yanagisawa, S., Yoo, S., and Sheen, J. (2003). Differential regulation of EIN3 stability by glucose and ethylene signalling in plants. Nature 425, 521-525. doi: 10.1038 /nature01984

Yang, W., Liu, J., Tan, Y., Zhong, S., Tang, N., Chen, G., et al. (2015). Functional characterization of PhGR and PhGRL1 during flower senescence in the petunia. Plant Cell Rep. 34, 1561-1568. doi: 10.1007/s00299-0151808-7

Yang, Y., Wu, Y., Pirrello, J., Regad, F., Bouzayen, M., Deng, W., et al. (2009). Silencing S1-EBF1 and Sl-EBF2 expression causes constitutive ethylene response phenotype, accelerated plant senescence, and fruit ripening in tomato. J. Exp. Bot. 61, 697-708. doi: 10.1093/jxb/erp332

Conflict of Interest Statement: The authors declare that the research was conducted in the absence of any commercial or financial relationships that could be construed as a potential conflict of interest.

Copyright (c) $2016 \mathrm{Liu}, \mathrm{Hu}, \mathrm{Cai}, \mathrm{Lin}, \mathrm{Liu}$ and Yu. This is an open-access article distributed under the terms of the Creative Commons Attribution License (CC BY). The use, distribution or reproduction in other forums is permitted, provided the original author(s) or licensor are credited and that the original publication in this journal is cited, in accordance with accepted academic practice. No use, distribution or reproduction is permitted which does not comply with these terms. 